\title{
Fluorose dentária em crianças de Princesa Isabel, Paraíba
}

\section{Dental fluorosis in children from Princesa Isabel, Paraíba}

\author{
Franklin Delano Soares FORTE* \\ Claudia Helena Soares Morais FREITAS** \\ Fábio Correia SAMPAIO** \\ Maria Carmen de Araújo Melo JARDIM**
}

\begin{abstract}
FORTE, F. D. S.; FREITAS, C. H. S. M.; SAMPAIO, F. C.; JARDIM, M. C. de A. M. Fluorose dentária em crianças de Princesa Isabel, Paraíba. Pesqui Odontol Bras, v. 15, n. 2, p. 87-90, abr./jun. 2001.

Diversas localidades da Paraíba possuem níveis moderados ou elevados de fluoreto in natura nas águas de abastecimento. Nas áreas onde os níveis de fluoretos são considerados "ótimos" para a região (0,6 ppm), já constatou-se uma moderada prevalência de fluorose dentária (30-40\%). O presente trabalho teve por objetivo observar a prevalência de fluorose dentária na cidade de Princesa Isabel, com níveis "subótimos" de fluoretos $(0,4$ ppm). Foram selecionados aleatoriamente 142 escolares de 10 a 15 anos para o levantamento de fluorose dentária pelo índice TF (de Thylstrup e Fejerskov). Os exames foram realizados por três examinadores previamente calibrados, sob luz natural indireta e após escovação supervisionada e secagem dos elementos dentários. Cerca de $20 \%$ dos escolares examinados apresentaram fluorose dentária, sendo que $70 \%$ com TF 1 e outros $30 \%$ distribuídos em diversos graus (TF 2 a 5). Fluorose foi mais prevalente no sexo masculino e em dentes pré-molares. Embora a fluorose dentária observada esteja dentro dos níveis esperados para o teor de fluoretos $(0,4 \mathrm{ppm})$, outras fontes sistêmicas de fluoretos devem ser controladas. A prevalência de fluorose dentária observada não é problema de saúde pública nesta localidade.
\end{abstract}

UNITERMOS: Flúor; Fluorose dentária.

\section{INTRODUÇÃO}

Os fluoretos podem produzir efeitos adversos de forma crônica ou aguda. Na crônica, os fluoretos circulantes no organismo afetam a mineralização dos dentes formando um esmalte hipoplásico de diferentes manifestações clínicas que se denominou fluorose dentária ${ }^{6,7,18}$. Clinicamente, a fluorose dentária caracteriza-se por apresentar esmalte opaco e manchas de coloração que podem variar do branco ao marrom escuro, como também por apresentar áreas hipoplásicas e de erosões ${ }^{5,11}$. A severidade dessa condição do esmalte depende, segundo VILLENA; CURY ${ }^{18}$ (1998) da dose, duração, exposição, estágio da atividade dos ameloblastos, da idade do individuo e da susceptibilidade individual.

A concentração de fluoretos na água de abastecimento, considerada como "ótima" para a prevenção da cárie, é de 0,7-1,2 ppm conforme a temperatura média anual, podendo provocar o mínimo de fluorose dentária ${ }^{3}$. Em zonas tropicais, onde altas temperaturas levam a uma maior ingestão de líquidos, a concentração de fluoretos recomendada pode ser ainda mais baixa variando em torno de 0,5 a $0,7 \mathrm{ppm}^{2,19}$.

Apesar dos diferentes índices utilizados para registrar fluorose dentária, recentes estudos observaram que a prevalência de fluorose em diversas zonas urbanizadas do país está abaixo de 30\% para cidades com 0,8 ppm F na água de consumo ${ }^{1,4,9,16}$. Uma prevalência acima deste percentual tem sido atribuída ao efeito combinado de produtos dentários e fluoretos em águas de abastecimento ${ }^{17}$. A maioria desses estudos epidemiológicos é realizada em cidades com fluoretação artificial nas regiões Sul, Sudeste e Centro-Oeste do país. No Nordeste, poucos estudos exploraram a questão de fluorose dentária, embora existam fontes de água para consumo humano com teores elevados de fluoretos de forma natural. A fluorose dentária na Paraíba varia em torno de $31 \%$ em áreas com 0,4 a 0,7 ppm, 61\% em áreas com 0,8 a 1,0 ppm e $71 \%$ em áreas com fluoreto em águas de consumo acima de $1,0 \mathrm{ppm}^{13}$.

O presente levantamento tem como objetivo observar a prevalência de fluorose dentária em escolares de Princesa Isabel, Paraíba.

\footnotetext{
* Mestre em Odontologia, Área de Concentração em Odontopediatria, Universidade Federal de Santa Catarina.

** Professores da Disciplina de Odontologia Preventiva, Departamento de Clínica e Odontologia Social, Centro de Ciências da Saúde, Universidade Federal da Paraíba.
} 
FORTE, F. D. S.; FREITAS, C. H. S. M.; SAMPAIO, F. C.; JARDIM, M. C. de A. M. Fluorose dentária em crianças de Princesa Isabel, Paraíba. Pesqui Odontol Bras, v. 15, n. 2, p. 87-90, abr./jun. 2001.

\section{METODOLOGIA}

A cidade de Princesa Isabel possui 22.242 habitantes, e está localizada a $460 \mathrm{~km}$ da capital João Pessoa na zona fisiográfica do Sertão paraibano (IBGE, 1999) ${ }^{8}$. A temperatura média anual fica em torno de $25^{\circ}$ e $33^{\circ} \mathrm{C}$.

Foram selecionados aleatoriamente e examinados 142 escolares (10 a 15 anos) de ambos os sexos, matriculados em escolas públicas. A seleção obedeceu também ao critério de residência permanente, ou seja, as crianças deveriam ter nascido e sempre morado em Princesa Isabel. Até a data deste levantamento, nenhum programa de fluoretação tópica ou sistêmica havia sido implementado nestas escolas.

O exame foi realizado nas próprias escolas, sob iluminação natural indireta, por três dentistas, previamente calibrados (indice kappa considerado satisfatório de aproximadamente 0,80), utilizando espelho bucal, sonda exploradora, espátula de madeira, mesa e cadeiras. Previamente ao exame, as crianças realizaram higiene bucal com escova dental e dentifrícios, sob a supervisão de um profissional. Os dentes foram mantidos secos por pelo menos 30 segundos com auxílio de gaze.

$\mathrm{O}$ índice de fluorose dentária utilizado foi o $\mathrm{TF}$ que foi proposto por THYLSTRUP; FEJERSKOV ${ }^{16}$ (1978). Consideraram-se as faces vestibulares de todos os dentes permanentes, entretanto não foram considerados os elementos dentários comprometidos por processos cariosos extensos que impossibilitassem o diagnóstico, dentes não irrompidos completamente, dentes hipoplásicos com amelogênese e dentinogênese imperfeita e hipocalcificação, cuja causa excluísse seguramente a possibilidade da ingestão excessiva de flúor. Para cada dente, foi obtido o índice, tendo sido registrado em uma ficha clínica individual.

Três amostras da água de abastecimento da cidade foram coletadas na época da realização dos exames: em uma residência; em uma das escolas e na sede da Companhia de Águas e Esgotos da Paraíba (CAGEPA). As análises de fluoretos foram realizadas no Laboratório de Bioquímica da $\mathrm{Fa}-$ culdade de Odontologia da UNICAMP, que demonstrou haver 0,4 ppm de flúor nas 3 amostras coletadas.

\section{RESULTADOS}

Fluorose dentária foi observada em $20 \%$ da população estudada $(n=29)$, sendo a maioria dos casos TF $1(n=20)$. No restante da amostra, observaram-se índices de TF 2 até no máximo 5.

A Tabela 1 apresenta a distribuição dos elementos dentários por diferentes graus de fluorose. $\mathrm{Na}$ Tabela 2, visualizam-se os diferentes graus de fluorose por grupos dentários.

TABELA 1 - Distribuição de fluorose dentária (TF 1 a 5) em 142 crianças de ambos os sexos residentes permanentes em Princesa Isabel, PB.

\begin{tabular}{l|c|c|c|c|c|c|c|c}
\hline \hline \multirow{2}{*}{ Sexo } & \multirow{2}{*}{$\mathrm{N}$} & \multirow{2}{*}{ Dentes } & \multicolumn{6}{|c}{ Grau de fluorose (TF)* } \\
\cline { 4 - 9 } & & & 0 & 1 & 2 & 3 & 4 & 5 \\
\hline Feminino & 73 & 2.044 & $1.693(83)$ & $271(13)$ & $55(3)$ & $24(1)$ & 0 & $1(0,05)$ \\
\hline Masculino & 69 & 1.932 & $1.468(76)$ & $288(15)$ & $155(8)$ & $11(0,5)$ & $8(0,4)$ & $2(0,1)$ \\
\hline Total & 142 & 3.976 & $3.161(80)$ & $559(14)$ & $210(5)$ & $35(1)$ & $8(0,2)$ & $2(0,05)$ \\
\hline \hline
\end{tabular}

*Percentuais em parênteses.

TABELA 2 - Distribuição percentual de fluorose dentária com diferentes graus e severidade por grupo dentário em crianças residentes permanentes em Princesa Isabel, PB.

\begin{tabular}{|c|c|c|c|c|c|c|}
\hline \multirow{2}{*}{ Grupo dentário } & \multicolumn{6}{|c|}{ Grau de fluorose dentária (TF) \% } \\
\hline & 0 & 1 & 2 & 3 & 4 & 5 \\
\hline Incisivos & 86,7 & 8,5 & 3,9 & 0,5 & 0,1 & 0,08 \\
\hline Caninos & 79,4 & 14,9 & 5,1 & 0,5 & 0 & 0 \\
\hline Pré-molares & 66,1 & 24,2 & 7,6 & 1,5 & 0,2 & 0,08 \\
\hline Molares & 85,7 & 8,9 & 4,3 & 0,6 & 0,2 & 0,08 \\
\hline
\end{tabular}


FORTE, F. D. S.; FREITAS, C. H. S. M.; SAMPAIO, F. C.; JARDIM, M. C. de A. M. Fluorose dentária em crianças de Princesa Isabel, Paraíba. Pesqui Odontol Bras, v. 15, n. 2, p. 87-90, abr./jun. 2001.

\section{DISCUSSÃO}

A prevalência de fluorose dentária em $20 \%$ das crianças de Princesa Isabel está dentro dos valores esperados para o nível de fluoretos na água de abastecimento $(0,4 \mathrm{ppm})$. Este resultado está ligeiramente abaixo dos valores encontrados por SILVA; PAIVA ${ }^{15}$ (1995) e ALCÂNTARA ${ }^{1}$ (1998) que, usando o índice de Dean, observaram uma prevalência de $25 \%$ em Belo Horizonte e Curitiba, respectivamente. Além da diferença dos índices de fluorose utilizados, vale salientar que estas cidades possuem fluoretação artificial em 0,75 ppm, praticamente o dobro do nivel de fluoretos de Princesa Isabel. Utilizando o indice TF, VALOIS et al. ${ }^{17}$ (1999) e MALTZ; SILVA $^{9}$ (2000) observaram uma prevalência de fluorose em torno de $34 \%$ e $49 \%$, respectivamente, em cidades com fluoretação artificial em torno de 0,8 ppm. A baixa prevalência de fluorose (15\%) observada por CAMPOS ${ }^{4}$ (1998) em Brasília $(0,8 \mathrm{ppm})$ pode ser em parte explicada pela diferença de índices utilizados. Além disso, fatores como a variação no uso e ingestão de dentifrícios fluoretados dificultam comparações entre esses levantamentos epidemiológicos. No entanto, todos esses estudos encontraram com mais freqüência as formas mais leves de fluorose dentária. Portanto, uma freqüência maior no indice TF 1 também era previsto para Princesa Isabel.

No estudo de SAMPAIO et al. ${ }^{13}$ (1999), realizado em áreas com fluoretos entre 0,4 e 0,7 ppm na $\mathrm{Pa}-$ raíba, observaram-se $31 \%$ de fluorose pelo índice $\mathrm{TF}$ - uma prevalência de fluorose $10 \%$ acima da observada no presente estudo. Estes valores são provavelmente causados pela diferença de concentrações de fluoretos na água de consumo das comunidades pesquisadas. A ingestão de dentifrício fluoretado seria de menor importância, pois o uso de dentifrícios por pré-escolares e escolares nas escolas públicas do interior da Paraíba ainda é relativamente limitado ${ }^{13}$. Além disso, como já observado em outras partes do país, o uso de dentifrícios fluoretados é possivelmente mais restrito nas escolas públicas do que nas escolas particulares $^{9,12}$.
Quanto aos grupos dentários estudados, a prevalência de fluorose observada no presente estudo foi maior nos pré-molares, semelhante aos dados observados por VALOIS et al. ${ }^{17}$ (1999), mas diferente dos dados de PAIVA; BARROS FILHO ${ }^{10}$ (1993) que pesquisaram fluorose em áreas com elevadas concentrações de fluoretos nas águas de abastecimento (1,2 até $5,6 \mathrm{ppm})$.

Em relação ao sexo, houve uma discreta diferença (Tabela 1), sendo no masculino a prevalência maior que no feminino. Semelhante aos estudos de CAPELLA $^{5}$ (1989) e CAMPOS ${ }^{4}$ (1998), esta diferença não foi significativa estatisticamente (MannWhitney U, p > 0,01).

Casos de fluorose com comprometimento estético (TF 3, 4 e 5) em comunidades do interior da Paraíba são relativamente freqüentes ${ }^{14}$. Apesar de o teor de fluoretos na água de abastecimento de Princesa Isabel estar abaixo do que seria considerado o nível "ótimo", observaram-se alguns casos de fluorose com necessidade de tratamento. Portanto, o controle de fontes externas de fluoretos em áreas com valores "subótimos" é recomendável, particularmente em regiões de clima quente onde o consumo de líquidos é elevado e há conseqüentemente um maior risco de fluorose dentária. Uma maior disponibilidade de produtos fluoretados em algumas áreas do interior da Paraíba já está sendo observada ${ }^{12}$. Estudos sobre a ingestão de fluoretos são necessários para um adequado monitoramento da fluorose dentária nestas comunidades.

\section{CONCLUSÕES}

Observou-se uma prevalência de fluorose dentária em cerca de $20 \%$ da população estudada, sendo ligeiramente maior no sexo masculino do que no feminino.

Em relação aos grupos dentários, fluorose foi mais freqüente nos pré-molares (aproximadamente $34 \%)$, seguidos dos caninos (21\%), incisivos e molares (14\%).

Os dados observados sugerem que a fluorose dentária não é problema de saúde pública em escolares de Princesa Isabel $(0,4 \mathrm{ppm})$. De qualquer modo, outras formas sistêmicas de fluoretos devem ser controladas. 
FORTE, F. D. S.; FREITAS, C. H. S. M.; SAMPAIO, F. C.; JARDIM, M. C. de A. M. Fluorose dentária em crianças de Princesa Isabel, Paraíba. Pesqui Odontol Bras, v. 15, n. 2, p. 87-90, abr./jun. 2001.

FORTE, F. D. S.; FREITAS, C. H. S. M.; SAMPAIO, F. C.; JARDIM, M. C. de A. M. Dental fluorosis in children from Princesa Isabel, Paraíba. Pesqui Odontol Bras, v. 15, n. 2, p. 87-90, abr./jun. 2001.

Several communities in Paraiba have moderate or high levels of fluoride naturally present in the drinking water. A moderate prevalence of dental fluorosis (30-40\%) has been observed in some areas where the levels of fluoride are regarded as "optimal" for the region $(0.6 \mathrm{ppm})$. The aim of the present study was to determine the prevalence of dental fluorosis in Princesa Isabel, a city with "sub-optimal" fluoride levels (0.4 ppm). The sample comprised 142 schoolchildren (10- to 15-year-old subjects) randomly selected and examined by means of the TF (Thylstrup \& Fejerskov) index. The clinical exams were carried out under indirect natural light by three calibrated examiners. Prior to the examination the teeth were cleaned and dried. Approximately $20 \%$ of the subjects examined presented with some degree of fluorosis. Seventy per cent were classified as TF 1 while $30 \%$ were classified as TF 2 to 5 . The prevalence of fluorosis was higher in male subjects and in premolars. Although the observed prevalence of dental fluorosis was within the expected levels, other sources of systemic fluoride must be controlled. The observed prevalence of dental fluorosis is not a public health problem in this community.

UNITERMS: Fluorine; Dental fluorosis.

\section{REFERÊNCIAS BIBLIOGRÁFICAS}

1. ALCÂNTARA, C. M. Prevalência de fluorose dental em escolares de Curitiba. Rev ABO Nac, v. 6, n. 5, p. 304307, 1998.

2. BROUWER, I. D.; de BRUIN, A.; HAUTVAST, J. G. et al. Unsuitability of the World Health Organization guidelines for fluoride concentrations in drinking water in Senegal. Lancet, v. 1, n. 8.579, p. 223-225, 1988.

3. BUENDIA, O. C.; ZAINA, S. Fluorose dentária na cidade de São João do Pau d'Alho. Rev Ass Paul Cir Dent, v. 36, n. 6, p. 625, nov./dez. 1982.

4. CAMPOS, D. L. Prevalência de fluorose dentária em escolares de Brasília, Distrito Federal. Rev Odonto Univ São Paulo, v. 12, n. 3, p. 225-230, 1998.

5. CAPELLA, L. F. Ocorrência de fluorose dentária endêmica. RGO, v. 37, n. 5, p. 371-375, 1989.

6. CURY, J. A. Manipulando flúor com segurança. Parte III toxicidade crônica. J Aboprev, ano 6, jan./fev./mar. 1995.

7. FEJERSKOV, O.; MANJI, F.; BAELUM, V. et al. Fluorose dentária: manual para profissionais de saúde. Santos : São Paulo, 1994. 122 p.

8. IBGE: Instituto Brasileiro de Geografia e Estatística. Disponivel na internet: $h t t p: \backslash \backslash w w w . i b g e . g o v . b r$. 22/08/1999.

9. MALTZ, M.; SILVA, B. B. Relationship of caries, gingivitis and fluorosis to socioeconomic status in Brazilian 12-year-old schoolchildren. $47^{\text {th }}$ Orca Congress, Alghero, Italy, 2000. Caries Res, n. 34, p. 315-316, 2000. [Resumo n. 22].

10. PAIVA, S. M.; BARROS FILHO, M. A. Contribuição ao estudo da fluorose dentária, na dentição permanente, numa comunidade com fluorose endêmica (Cocal, Urussanga, Santa Catarina). Rev Odontopediatria, v. 2, n. 1, p. 5-15, 1993.
11. PINBORG, J. J. Pathology of the dental hard tissues. Copenhagen : Munksgaard, 1970.

12. SAMPAIO, F. C.; NAZMUL HOSSAIN, A. N. M.; von der FEHR, F. R. et al. Dental caries and sugar intake of children from rural areas with different water fluoride levels in Paraíba, Brazil. Community Dent Oral Epidemiol, n. 28, p. 307-13, 2000.

13. SAMPAIO, F. C.; von der FEHR, F. R.; ARNEBERG, P. et al. Dental fluorosis and nutritional status of 6- to 11-year-old children living in rural areas of Paraíba, Brazil. Caries Res, n. 33, p. 666-73, 1999.

14. SAMPAIO, F. C.; MENDES, A. C. R.; PIRES, G. R. Remoção de manchas de fluorose pela técnica de Croll: descrição de caso clínico. Rev ABO Nac, n. 5, p. 153-56, 1997.

15. SILVA, A. L. C. C.; PAIVA, S. M. Ocorrência de fluorose dentária em escolares de Belo Horizonte. Rev CROMG, v. 1, n. 2, p. 49-53, 1995.

16. THYLSTRUP, A.; FEJERSKOV, O. Clinical appearance of dental fluorosis in permanent teeth in relation to histological changes. Community Dent Oral Epidemiol, v. 6, p. 315-328, 1978.

17. VAlOIS, C. A.; SOVIERO, V. M.; CRUZ, R. A. Avaliação do grau de fluorose dental em crianças de 7 a 12 anos de idade. $16^{a}$ REUNIÃO ANUAL DA SOCIEDADE BRASILEIRA DE PESQUISA ODONTOLÓGICA, 1999, Águas de São Pedro. Anais. 1999. [Resumo B-109].

18. VILLENA, R. S.; CURY, J. A. Flúor: aplicação sistêmica. In: CORREIA, M. S. N. P. Odontologia para a primeira infância. São Paulo: Santos, 1998. 291-314 p.

19. WARNAKULASURIYA, K. A.; BALASURIYA, S.; PERERA, P. A. et al. Determining optimal levels of fluoride in drinking-water for hot, dry climates: a case study in Sri Lanka. Community Dent Oral Epidemiol, v. 20, p. 364-367, 1992.
Recebido para publicação em 16/08/00 Enviado para reformulação em 09/02/01 Aceito para publicação em 10/04/01 